\title{
New Existence Results for Fixed Point Problem and Minimization Problem in Compact Metric Spaces
}

\author{
Weng-Seng Heng, ${ }^{1}$ Wei-Shih Du, ${ }^{2}$ and Chi-Lin Yen $^{3}$ \\ ${ }^{1}$ Department of Applied Mathematics, Chinese Culture University, Taipei 11114, Taiwan \\ ${ }^{2}$ Department of Mathematics, National Kaohsiung Normal University, Kaohsiung 82444, Taiwan \\ ${ }^{3}$ Department of Mathematics, National Taiwan Normal University, Taipei 11677, Taiwan
}

Correspondence should be addressed to Wei-Shih Du; wsdu@nknucc.nknu.edu.tw

Received 24 April 2014; Accepted 4 June 2014; Published 16 June 2014

Academic Editor: Erdal Karapınar

Copyright (c) 2014 Weng-Seng Heng et al. This is an open access article distributed under the Creative Commons Attribution License, which permits unrestricted use, distribution, and reproduction in any medium, provided the original work is properly cited.

We first present some new existence theorems for fixed point problem and minimization problem in compact metric spaces without assuming that mappings possess convexity property. Some applications of our results to new fixed point theorems for nonself mappings in the setting of strictly convex normed linear spaces and usual metric spaces are also given.

\section{Introduction and Preliminaries}

Let $(X, d)$ be a metric space. Denote by $\mathscr{N}(X)$ the family of all nonempty subsets of $X$. The symbols $\mathbb{N}, \mathbb{R}$, and $\mathbb{C}$ are used to denote the sets of positive integers, real numbers, and complex numbers, respectively. Let $K$ be a nonempty subset of $X$, let $T: K \rightarrow X$ be a single-valued mapping, and let $S$ : $K \rightarrow \mathcal{N}(X)$ be a multivalued mapping. A point $v$ in $K$ is said to be a fixed point of $T$ (resp. $S$ ) if $T v=v$ (resp. $v \in S v$ ). The set of fixed points of $T$ (resp. $S$ ) is denoted by $\mathscr{F}(T)($ resp. $\mathscr{F}(S))$. An extended real valued function $f: X \rightarrow(-\infty,+\infty]$ is said to be lower semicontinuous at $\hat{x} \in X$ if, for any sequence $\left\{x_{n}\right\}$ in $X$ with $x_{n} \rightarrow \widehat{x}$, we have $f(\widehat{x}) \leq \liminf _{n \rightarrow \infty} f\left(x_{n}\right)$. The function $f$ is called to be lower semicontinuous on $X$ if $f$ is lower semicontinuous at every point of $X$. The function $f$ is said to be proper if $f \neq \equiv \infty$.

Let $(E,\|\cdot\|)$ be a normed linear space over the field $\mathbb{K}=$ $\mathbb{C}$ or $\mathbb{R} . E$ is said to be strictly convex if $x=y$ whenever $\|(x+y) / 2\|=\|x\|=\|y\|$; in other words, the unit sphere of $E$ does not contain nontrivial segments. It is worth mentioning that the strict convexity of a normed linear space $E$ can be characterized by the properties: for any nonzero vectors $x, y \in E$, if $\|x+y\|=\|x\|+\|y\|$, then $y=c x$ for some real $c>0$. The following four types of line segments between two distinct points $a$ and $b$ of $E$ are defined as the sets:

$$
\begin{aligned}
& \operatorname{Seg}(a, b)=\{\lambda a+(1-\lambda) b: \lambda \in(0,1)\}, \\
& \operatorname{Seg}[a, b)=\{\lambda a+(1-\lambda) b: \lambda \in(0,1]\}, \\
& \operatorname{Seg}(a, b]=\{\lambda a+(1-\lambda) b: \lambda \in[0,1)\}, \\
& \operatorname{Seg}[a, b]=\{\lambda a+(1-\lambda) b: \lambda \in[0,1]\} .
\end{aligned}
$$

Clearly, $\operatorname{Seg}[a, b]$ is a closed subset of $E$.

The celebrated Banach contraction principle [1] plays an important role in various fields of nonlinear analysis and applied mathematical analysis.

Theorem 1 (Banach [1]). Let $(X, d)$ be a complete metric space and let $T: X \rightarrow X$ be a selfmap. Assume that there exists a nonnegative number $\gamma<1$ such that

$$
d(T(x), T(y)) \leq \gamma d(x, y) \quad \forall x, y \in X .
$$

Then T has a unique fixed point in X. Moreover, for each $x \in X$, the iterative sequence $\left\{T^{n} x\right\}_{n \in \mathbb{N}}$ converges to the unique fixed point of $T$. 
Let $K$ be a nonempty subset of a metric space $(X, d)$ and let $T: K \rightarrow K$ be a mapping. Recall that $T$ is said to be contractive [2] if

$$
d(T x, T y)<d(x, y) \quad \forall x, y \in K \text { with } x \neq y .
$$

The following interesting fixed point theorem in the setting of compact metric spaces is due to Edelstein in [2].

Theorem 2 (Edelstein [2]). Let $(K, d)$ be a nonempty compact metric space and let $T: K \rightarrow K$ be contractive. Then $T$ has a unique fixed point in $K$.

In 1976, Caristi proved the following famous fixed point theorem to extend Banach contraction principle.

Theorem 3 (Caristi [30]). Let $(X, d)$ be a complete metric space and let $f: X \rightarrow \mathbb{R}$ be a lower semicontinuous and bounded below function. Suppose that $T$ is a Caristi-type map on $X$ dominated by $f$; that is, $T$ satisfies

$$
d(x, T x) \leq f(x)-f(T x) \quad \text { for each } x \in X
$$

Then $T$ has a fixed point in $X$.

It is well-known that Caristi's fixed point theorem is equivalent to Ekeland's variational principle, to Takahashi's nonconvex minimization theorem, to Daneš' drop theorem, to petal theorem, and to Oettli-Théra's theorem; see, for example, $[3,4]$ and references therein for more details. In view of the important contribution of Caristi's fixed point theorem on nonlinear analysis, a great deal of generalizations in various different directions of the Caristi's fixed point theorem has been investigated by several authors. For more details on these generalizations, one can refer to [3-19] and references therein.

During the last few decades, an interesting and important direction of research in metric fixed point theory is to study the existence and uniqueness of fixed points for single-valued nonself mappings or multivalued nonself mappings satisfying certain nonlinear conditions. A mass of such research has been investigated by many authors; see, for example, [20-29] and the references therein.

In this work, we first present some new existence theorems for fixed point problem and minimization problem in compact metric spaces without assuming that mappings possess convexity property. Some applications of our results to new fixed point theorems for nonself mappings in the setting of strictly convex normed linear spaces and usual metric spaces are also given.

\section{Existence Results for Fixed Point Problem and Minimization Problem without Convexity}

We start with the following crucial and useful existence result for fixed point problem and minimization problem which is one of the main results of this paper.
Theorem 4. Let $(K, d)$ be a nonempty compact metric space, let $f: K \rightarrow(-\infty,+\infty]$ be a proper lower semicontinuous function bounded from below, and let $T: K \rightarrow \mathcal{N}(K)$ be a multivalued mapping. Suppose that

(H1) for any $x \in K$ with $x \notin T x$, there exists $y \in T x$ such that

$$
f(y)<f(x) .
$$

Then, there exists $v \in K$ such that

(a) $v \in T v$,

(b) $f(v)=\inf \{f(x): x \in T v\}=\inf \{f(x): x \in K\}<$ $+\infty$.

Proof. Since $f$ is bounded from below,

$$
c:=\inf \{f(x): x \in K\}>-\infty .
$$

Since $f$ is proper, there exists $w \in K$ such that $f(w)<+\infty$. It follows that

$$
c \leq f(w)<+\infty .
$$

Hence, by (6) and (7), we know $c \in \mathbb{R}$. One can find a sequences $\left\{v_{n}\right\}_{n \in \mathbb{N}}$ in $K$ such that

$$
\lim _{n \rightarrow \infty} f\left(v_{n}\right)=c
$$

By the compactness of $K$, there exists subsequences $\left\{v_{n_{j}}\right\} \subset$ $\left\{v_{n}\right\}$ and $v \in K$ such that

$$
v_{n_{j}} \longrightarrow v \text { as } j \longrightarrow \infty \text {. }
$$

By the lower semicontinuity of $f$ and (8), we have

$$
c \leq f(v) \leq \liminf _{j \rightarrow \infty} f\left(v_{n_{j}}\right)=c,
$$

which implies

$$
f(v)=c=\inf \{f(x): x \in K\} .
$$

Next, we claim that $v \in T v$. On the contrary, assume that $v \notin T v$. Then, by our hypothesis $(H 1)$, there exists $y_{v} \in T v$ such that

$$
f(v)>f\left(y_{v}\right) \geq f(v),
$$

which is a contradiction. Therefore $v \in T v$ and the conclusion (a) is proved. Due to

$$
f(v)=\inf \{f(x): x \in K\} \leq \inf \{f(x): x \in T v\} \leq f(v),
$$

we show the conclusion (b). The proof is completed.

The following existence theorem is obviously an immediate result from Theorem 4 . 
Theorem 5. Let $(K, d)$ be a nonempty compact metric space, let $f: K \rightarrow(-\infty,+\infty]$ be a proper lower semicontinuous function bounded from below, and let $T: K \rightarrow K$ be a singlevalued selfmapping. Suppose that

(H2) $f(x)-f(T x)>0$ for any $x \in K$ with $x \neq T x$.

Then there exists $v \in K$ such that

(a) $T v=v$,

(b) $f(v)=\inf \{f(x): x \in K\}<+\infty$.

In fact, we have the following important fact.

Theorem 6. Theorems 4 and 5 are equivalent.

Proof. It suffices to show that Theorem 5 implies Theorem 4. Under the assumption (H1) of Theorem 4 , for any $x \in K$ with $x \notin T x$, there exists $y_{x} \in T x$ such that

$$
f\left(y_{x}\right)<f(x) .
$$

So, we can define a single-valued selfmap $G: K \rightarrow K$ by

$$
G x:= \begin{cases}x, & \text { if } x \in T x \\ y_{x}, & \text { if } x \notin T x .\end{cases}
$$

It is easy to see that $G$ satisfies $f(x)-f(G x)>0$ for any $x \in K$ with $x \neq G x$. So, all the hypotheses of Theorem 5 are fulfilled. It is therefore possible to apply Theorem 5 to get $v \in K$ such that

(a) $G v=v$,

(b) $f(v)=\inf \{f(x): x \in K\}<+\infty$.

By (a) and the definition of $G$, we have $v \in T v$. From (b) and $v \in T v$, we get

$$
f(v)=\inf \{f(x): x \in T v\}=\inf \{f(x): x \in K\}<+\infty .
$$

Therefore Theorem 5 implies Theorem 4 and hence the proof is completed.

Applying Theorem 4, we establish the following compactness version of Caristi's type fixed point theorem for multivalued mappings.

Theorem 7. Let $(K, d)$ be a nonempty compact metric space, let $f: K \rightarrow(-\infty,+\infty)$ be a proper lower semicontinuous function bounded from below, and let $T: K \rightarrow \mathcal{N}(K)$ be a multivalued mapping. Suppose that, for any $x \in K$, there exists $y \in T x$ such that

$$
d(x, y) \leq f(x)-f(y)
$$

Then there exists $v \in K$ such that

(a) $v \in T v$,

(b) $f(v)=\inf \{f(x): x \in T v\}=\inf \{f(x): x \in K\}<$ $+\infty$.
Proof. For any $x \in K$ with $x \notin T x$, by our hypothesis, there exists $y \in T x$ such that

$$
0<d(x, y) \leq f(x)-f(y)
$$

which implies

$$
f(y)<f(x) .
$$

So $(H 1)$ as in Theorem 4 is satisfied. Therefore the conclusion follows from Theorem 4 .

As a direct consequence of Theorem 7 we obtain the following result which is a compactness version of Caristi's fixed point theorem.

Theorem 8. Let $(K, d)$ be a nonempty compact metric space, let $f: K \rightarrow(-\infty,+\infty]$ be a proper lower semicontinuous function bounded from below, and let $T: K \rightarrow K$ be a singlevalued selfmapping. Suppose that $T$ is a Caristi-type map on $K$ dominated by $f$; that is, $T$ satisfies

$$
d(x, T x) \leq f(x)-f(T x) \quad \text { for each } x \in K .
$$

Then there exists $v \in K$ such that

(a) $T v=v$,

(b) $f(v)=\inf \{f(x): x \in K\}<+\infty$.

Theorem 9. Theorems 7 and 8 are equivalent.

By applying Theorem 5 (or Theorem 4), we obtain the following new fixed point theorem for nonself mappings in metric spaces.

Theorem 10. Let $K$ be a nonempty compact subset of a metric space $(X, d)$ and let $S: K \rightarrow X$ be a continuous mapping. Suppose that

(H3) for any $x \in K$ with $x \neq S x$ there exists $y \in K \backslash\{x\}$ such that

$$
d(y, S y)<d(x, S x) .
$$

Then S admits a fixed point in K.

Proof. Define $f: K \rightarrow \mathbb{R}$ by

$$
f(x)=d(x, S x) \quad \text { for } x \in K .
$$

By the continuity of $S, f$ is continuous and bounded below by 0 . By the assumption (H3), for any $x \in K$ with $x \neq S x$, there exists $y_{x} \in K \backslash\{x\}$ such that

$$
f\left(y_{x}\right)=d\left(y_{x}, S y_{x}\right)<d(x, S x)=f(x),
$$

so we can define a single-valued selfmap $T: K \rightarrow K$ by

$$
T x:= \begin{cases}x, & \text { if } x=S x \\ y x, & \text { if } x \neq S x .\end{cases}
$$


For any $x \in K$ with $x \neq S x$, by (23) and the definition of $T$, we obtain

$$
f(x)-f(T x)>0 .
$$

Hence we prove that (H3) implies (H2) in Theorem 5. Applying Theorem 5, there exists $v \in K$ such that $T v=v$, which deduce $S v=v$. The proof is completed.

Remark 11. Edelstein's fixed point theorem [2] (i.e., Theorem 2) is a special case of Theorem 10. Indeed, since $T$ is contractive, it is easy to see that $T$ is continuous on $K$. For any $x \in K$ with $x \neq T x$, let $y=T x$. Then $y \in K \backslash\{x\}$ and

$$
\begin{aligned}
d(x, T x)-d(y, T y) & =d(x, T x)-d\left(T x, T^{2} x\right) \\
& >d(x, T x)-d(x, T x)=0 .
\end{aligned}
$$

Hence $(H 3)$ as in Theorem 10 is satisfied. Therefore the conclusion follows from Theorem 10.

\section{Some Applications of Theorem 10}

In this section, we study some applications of Theorem 10 to fixed point theory. We first establish a new fixed point theorem without assuming that nonself mappings possess convexity property in the setting of strictly convex normed linear spaces by exploiting Theorem 10 .

Theorem 12. Let $(E,\|\cdot\|)$ be a strictly convex normed linear space, let $K$ be a nonempty compact subset of $E$, and let $T$ : $K \rightarrow E$ be a continuous mapping. Suppose that

(H4) for any $x \in K$ with $x \neq T x$ there exists $y \in K \backslash\{x\}$ and $y \neq T x$ such that

$$
\begin{gathered}
\|x-T x\|=\|x-y\|+\|y-T x\|, \\
\|T x-T y\| \leq\|x-y\| .
\end{gathered}
$$

\section{Then $T$ admits a fixed point in $K$.}

Proof. We first claim that the condition $(P)$ holds, where

(P) for any $x \in K$ with $x \neq T x$ there exists $y \in K \backslash\{x\}$ such that $\|y-T y\|<\|x-T x\|$.

Indeed, let $x \in K$ with $x \neq T x$ be given. By (H4), there exists $y_{x} \in K \backslash\{x\}$ and $y_{x} \neq T x$ such that

$$
\begin{gathered}
\|x-T x\|=\left\|x-y_{x}\right\|+\left\|y_{x}-T x\right\|, \\
\left\|T x-T y_{x}\right\| \leq\left\|x-y_{x}\right\| .
\end{gathered}
$$

It follows that

$$
\begin{aligned}
\left\|y_{x}-T y_{x}\right\| & \leq\left\|y_{x}-T x\right\|+\left\|T x-T y_{x}\right\| \\
& \leq\left\|y_{x}-T x\right\|+\left\|x-y_{x}\right\| \\
& =\|x-T x\| .
\end{aligned}
$$

If $\left\|y_{x}-T y_{x}\right\|<\|x-T x\|$, then our claim $(P)$ is finished.
Suppose $\left\|y_{x}-T y_{x}\right\|=\|x-T x\|$. Since $E$ is strictly convex, $x \neq y_{x}, y_{x} \neq T x$, and $\|x-T x\|=\left\|x-y_{x}\right\|+\left\|y_{x}-T x\right\|$, there exists $\lambda>0$ such that

$$
y_{x}-T x=\lambda\left(x-y_{x}\right) .
$$

Let $c=\lambda /(1+\lambda)$. Then $c \in(0,1)$. By $(30)$, we have

$$
y_{x}=c x+(1-c) T x \in \operatorname{Seg}(x, T x) .
$$

Hence $y_{x} \in \operatorname{Seg}(x, T x) \cap K$. Put

$$
\mathscr{A}=\{w \in \operatorname{Seg}(x, T x) \cap K:\|w-T w\|=\|x-T x\|\} .
$$

Since $y_{x} \in \mathscr{A}, \mathscr{A} \neq \emptyset$. Let $\rho:=\sup _{w \in \mathscr{A}}\|w-x\|$. Then

$$
0<\left\|y_{x}-x\right\| \leq \rho<\sup _{w \in K}\|w-x\|<\infty .
$$

We can choose a sequence $\left\{v_{n}\right\} \subset \mathscr{A}$, such that

$$
\lim _{n \rightarrow \infty}\left\|v_{n}-x\right\|=\rho \text {. }
$$

Since $\left\{v_{n}\right\} \subset \operatorname{Seg}(x, T x) \cap K \subset \operatorname{Seg}[x, T x] \cap K$ and $\operatorname{Seg}[x, T x] \cap K$ is a nonempty compact subset of $E$, there exist a subsequence $\left\{v_{n_{i}}\right\}$ of $\left\{v_{n}\right\}$ and a vector $v \in \operatorname{Seg}[x, T x] \cap K$ such that

$$
v_{n_{i}} \longrightarrow v \quad \text { as } i \longrightarrow \infty \text {. }
$$

By taking into account (34) and (35), we get

$$
\|v-x\|=\lim _{i \rightarrow \infty}\left\|v_{n_{i}}-x\right\|=\rho>0,
$$

which implies $v \neq x$. So $v \in \operatorname{Seg}(x, T x] \cap K$ and hence there exists $\kappa \in[0,1)$ such that

$$
v=\kappa x+(1-\kappa) T x .
$$

On the other hand, by the continuity of $T$, we obtain

$$
T v_{n_{i}} \longrightarrow T v \quad \text { as } i \longrightarrow \infty .
$$

For any $i \in \mathbb{N}$, since $v_{n_{i}} \in \mathscr{A}$, we have

$$
\left\|v_{n_{i}}-T v_{n_{i}}\right\|=\|x-T x\| .
$$

Thus, by (37), we obtain

$$
\begin{aligned}
\|v-T v\| & \leq\left\|v-v_{n_{i}}\right\|+\left\|v_{n_{i}}-T v_{n_{i}}\right\|+\left\|T v_{n_{i}}-T v\right\| \\
& =\left\|v-v_{n_{i}}\right\|+\|x-T x\|+\left\|T v_{n_{i}}-T v\right\| \quad \forall i \in \mathbb{N} .
\end{aligned}
$$

By taking the limit from both sides of the last inequality, we get

$$
\|v-T v\| \leq\|x-T x\| .
$$

If $\|v-T v\|<\|x-T x\|$, then our claim $(P)$ is proved when we take $y=v$. Suppose $\|v-T v\|=\|x-T x\|$. Let

$$
\mathscr{B}=\{w \in \operatorname{Seg}[v, T x] \cap K:\|w-T w\|=\|v-T v\|\} .
$$


Then $\emptyset \neq \mathscr{B} \subset \mathscr{A}$. Let $\zeta:=\sup _{w \in \mathscr{B}}\|w-x\|$. Then $0<\zeta \leq \rho<$ $\infty$. We can find a sequence $\left\{z_{n}\right\} \subset \mathscr{B} \subset \operatorname{Seg}[v, T x] \cap K$, such that

$$
\lim _{n \rightarrow \infty}\left\|z_{n}-x\right\|=\zeta
$$

By the compactness of $\operatorname{Seg}[v, T x] \cap K$, there exist a subsequence $\left\{z_{n_{j}}\right\}$ of $\left\{z_{n}\right\}$ and a vector $z \in \operatorname{Seg}[v, T x] \cap K$ such that

$$
z_{n_{j}} \longrightarrow z \quad \text { as } j \longrightarrow \infty .
$$

From (43) and (44), we get

$$
\|z-x\|=\lim _{j \rightarrow \infty}\left\|z_{n_{j}}-x\right\|=\zeta .
$$

By (44) and the continuity of $T$, we have

$$
T z_{n_{j}} \longrightarrow T z \quad \text { as } j \longrightarrow \infty \text {. }
$$

For any $j \in \mathbb{N}$,

$$
\left\|z_{n_{j}}-T z_{n_{j}}\right\|=\|v-T v\|=\|x-T x\| \quad \text { due to } v_{n_{j}} \in \mathscr{B} .
$$

Since

$\|z-T z\| \leq\left\|z-z_{n_{j}}\right\|+\left\|z_{n_{j}}-T z_{n_{j}}\right\|+\left\|T z_{n_{j}}-T z\right\| \quad \forall j \in \mathbb{N}$,

taking into account (44), (46), and (47), we get

$$
\|z-T z\| \leq\|x-T x\| .
$$

We will verify $\|z-T z\|<\|x-T x\|$. Assume $\|z-T z\|=$ $\|x-T x\|=\|v-T v\|$. Then $z \in \mathscr{B}$. So $z=\eta v+(1-\eta) T x$ for some $\eta \in[0,1]$. Thus, by (37), we have

$$
\begin{aligned}
v & =\kappa x+(1-\kappa) T x \\
& =\kappa x+(1-\kappa)\left(\frac{1}{1-\eta} z-\frac{\eta}{1-\eta} v\right)
\end{aligned}
$$

which deduces

$$
v=\frac{\kappa(1-\eta)}{1-\eta \kappa} x+\frac{1-\kappa}{1-\eta \kappa} z .
$$

Since $\kappa \in[0,1)$, we have $1-\eta \kappa>0$ and $(1-\kappa) /(1-\eta \kappa)>0$. Because $\kappa(1-\eta) /(1-\eta \kappa)>0,(1-\kappa) /(1-\eta \kappa)>0$, and $\kappa(1-\eta) /(1-\eta \kappa)+(1-\kappa) /(1-\eta \kappa)=1$, we know $v \in \operatorname{Seg}(x, z)$ and hence

$$
\|x-z\|=\|x-v\|+\|v-z\| .
$$

Since $v \neq x$ and $v \neq z$, by (36), (45), and (52), we get

$$
\zeta=\|z-x\|>\|v-x\|=\rho,
$$

which leads a contradiction. Hence it must be $\|z-T z\|<$ $\|x-T x\|$. So our claim $(P)$ is proved when we take $y=z$. Now, all the hypotheses of Theorem 10 are fulfilled, so it is therefore possible to apply Theorem 10 to get the thesis.
As another interesting application of Theorem 10, we give the following new fixed point result for nonself mappings in usual metric spaces. It is worth mentioning that condition $(H 5)$ as in Theorem 13 is different from condition $(H 4)$ as in Theorem 12.

Theorem 13. Let $K$ be a nonempty compact subset of a metric space $(X, d)$ and let $S: K \rightarrow X$ be a continuous mapping. Suppose that

(H5) for any $x \in K$ with $x \neq S x$, there exists $y \in K \backslash\{x\}$ such that

$$
\begin{gathered}
d(x, y)+d(y, S x)=d(x, S x), \\
d(S x, S y)<d(x, y) .
\end{gathered}
$$

Then $S$ admits a fixed point in $K$.

Proof. Let $x \in K$ with $x \neq T x$ be given. Then, by (H5), there exists $y \in K \backslash\{x\}$ such that

$$
\begin{gathered}
d(x, y)+d(y, S x)=d(x, S x), \\
d(S x, S y)<d(x, y) .
\end{gathered}
$$

It follows from the last inequalities that

$$
\begin{aligned}
d(x, S x)-d(y, S y) & =d(x, y)+d(y, S x)-d(y, S y) \\
& \geq d(x, y)-d(S x, S y) \\
& >d(x, y)-d(x, y)=0 .
\end{aligned}
$$

So (H3) as in Theorem 10 is satisfied. Hence the conclusion follows from Theorem 10 .

Let $K$ be a nonempty subset of a metric space $(X, d)$. A mapping $T: K \rightarrow K$ is said to be metrically inward [30] if, for each $x \in K$, there exists $u \in K$ such that

$$
d(x, u)+d(u, T x)=d(x, T x)
$$

where $u=x$ if and only if $x=T x$.

Theorem 14. Let $K$ be a nonempty compact subset of a metric space $(X, d)$ and let $S: K \rightarrow X$ be a metrically inward contractive mapping. Then $S$ admits a unique fixed point in $K$.

Proof. Applying Theorem 13, $S$ has a fixed point in $K$. To see the uniqueness of fixed points of $S$, let $u, v \in \mathscr{F}(S)$. If $u \neq v$, since $S$ is contractive, we have

$$
d(u, v)=d(S u, S v)<d(u, v),
$$

a contradiction. Hence $u=v$ and $\mathscr{F}(S)$ is a singleton set. The proof is completed.

Finally, the following example is given to illustrate Theorem 14 . 
Example 15 (see [26, Example 3.1]). Let $X=\mathbb{R}^{2}$. Define a norm on $X$ by

$$
\|x\|=\max \left\{\left|x_{1}\right|,\left|x_{2}\right|\right\} \quad \text { for } x=\left(x_{1}, x_{2}\right) \in X .
$$

Then $(X,\|\cdot\|)$ is a Banach space and the norm is equivalent to the Euclidean norm on $X$. Let

$$
\begin{aligned}
K=\left\{\left(x_{1}, x_{2}\right) \in X: 0\right. & \leq x_{2} \leq x_{1}, \\
0 & \left.\leq x_{1} \leq \frac{1}{2}\right\} \cup\{(1-\sqrt{2}, 0)\} .
\end{aligned}
$$

So $K$ is a nonempty compact subset of $(X,\|\cdot\|)$. Define a mapping $S: K \rightarrow X$ by

$$
S x=\left(\frac{x_{1}^{2}-1}{2}, \frac{x_{2}^{2}}{2}\right) \quad \text { for } x=\left(x_{1}, x_{2}\right) \in K .
$$

Hence $S: K \rightarrow X$ is a metrically inward contractive mapping (see [26, Example 3.1]). By applying Theorem 14, we know that $S$ has a unique fixed point in $K$. In fact, precisely speaking, $(1-\sqrt{2}, 0)$ is the unique fixed point of $S$.

\section{Conflict of Interests}

The authors declare that there is no conflict of interests regarding the publication of this paper.

\section{Acknowledgment}

The second author was supported by Grant no. NSC 102-2115M-017-001 of the National Science Council of the Republic of China.

\section{References}

[1] S. Banach, "Sur les opérations dans les ensembles abstraits et leurs applications aux équations intégrales," Fundamenta Mathematicae, vol. 3, pp. 133-181, 1922.

[2] M. Edelstein, "An extension of Banach's contraction principle," Proceedings of the American Mathematical Society, vol. 12, pp. 7-10, 1961.

[3] D. H. Hyers, G. Isac, and T. M. Rassias, Topics in Nonlinear Analysis and Applications, World Scientific, Singapore, 1997.

[4] W. Takahashi, Nonlinear Functional Analysis, Yokohama Publishers, Yokohama, Japan, 2000.

[5] A. Petruşel and A. Sîntămărian, "Single-valued and multivalued Caristi type operators," Publicationes Mathematicae Debrecen, vol. 60, no. 1-2, pp. 167-177, 2002.

[6] O. Kada, T. Suzuki, and W. Takahashi, "Nonconvex minimization theorems and fixed point theorems in complete metric spaces," Mathematica Japonica, vol. 44, no. 2, pp. 381-391, 1996.

[7] T. Suzuki, "Generalized distance and existence theorems in complete metric spaces," Journal of Mathematical Analysis and Applications, vol. 253, no. 2, pp. 440-458, 2001.

[8] T. Suzuki, "Generalized Caristi’s fixed point theorems by Bae and others," Journal of Mathematical Analysis and Applications, vol. 302, no. 2, pp. 502-508, 2005.
[9] Y. Feng and S. Liu, "Fixed point theorems for multi-valued contractive mappings and multi-valued Caristi type mappings," Journal of Mathematical Analysis and Applications, vol. 317, no. 1, pp. 103-112, 2006.

[10] L.-J. Lin and W.-S. Du, "Ekeland's variational principle, minimax theorems and existence of nonconvex equilibria in complete metric spaces," Journal of Mathematical Analysis and Applications, vol. 323, no. 1, pp. 360-370, 2006.

[11] L.-J. Lin and W.-S. Du, "Some equivalent formulations of the generalized Ekeland's variational principle and their applications," Nonlinear Analysis: Theory, Methods \& Applications, vol. 67, no. 1, pp. 187-199, 2007.

[12] L.-J. Lin and W.-S. Du, "On maximal element theorems, variants of Ekeland's variational principle and their applications," Nonlinear Analysis: Theory, Methods \& Applications, vol. 68, no. 5, pp. 1246-1262, 2008.

[13] L.-J. Lin and W.-S. Du, "Systems of equilibrium problems with applications to new variants of Ekeland's variational principle, fixed point theorems and parametric optimization problems," Journal of Global Optimization, vol. 40, no. 4, pp. 663-677, 2008.

[14] W.-S. Du, "On some nonlinear problems induced by an abstract maximal element principle," Journal of Mathematical Analysis and Applications, vol. 347, no. 2, pp. 391-399, 2008.

[15] W.-S. Du, "On Caristi type maps and generalized distances with applications," Abstract and Applied Analysis, vol. 2013, Article ID 407219, 8 pages, 2013.

[16] W.-S. Du and E. Karapinar, "A note on Caristi-type cyclic maps: related results and applications," Fixed Point Theory and Applications, vol. 2013, article 344, 2013.

[17] K. Wodarczyk and R. Plebaniak, "Maximality principle and general results of ekeland and caristi types without lower semicontinuity assumptions in cone uniform spaces with generalized pseudodistances," Fixed Point Theory and Applications, vol. 2010, Article ID 175453, 2010.

[18] E. Karapinar, "Generalizations of Caristi Kirk's theorem on partial metric spaces," Fixed Point Theory and Applications, vol. 2011, article 4, 2011.

[19] W. A. Kirk and N. Shahzad, "Generalized metrics and Caristi's theorem," Fixed Point Theory and Applications, vol. 2013, article 129, 2013.

[20] F. E. Browder, "Nonexpansive nonlinear operators in a Banach space," Proceedings of the National Academy of Sciences of the United States of America, vol. 54, pp. 1041-1044, 1965.

[21] F. E. Browder and W. V. Petryshyn, "Construction of fixed points of nonlinear mappings in Hilbert space," Journal of Mathematical Analysis and Applications, vol. 20, pp. 197-228, 1967.

[22] W. A. Kirk, "Remarks on pseudo-contractive mappings," Proceedings of the American Mathematical Society, vol. 25, pp. 820823, 1970.

[23] W. A. Kirk, "Fixed point theorems for nonlinear nonexpansive and generalized contraction mappings," Pacific Journal of Mathematics, vol. 38, pp. 89-94, 1971.

[24] N. A. Assad and W. A. Kirk, "Fixed point theorems for set-valued mappings of contractive type," Pacific Journal of Mathematics, vol. 43, pp. 553-562, 1972.

[25] S. Reich, "Fixed points of condensing functions," Journal of Mathematical Analysis and Applications, vol. 41, pp. 460-467, 1973.

[26] X. P. Ding and Y. R. He, "Fixed point theorems for metrically weakly inward set-valued mappings," Journal of Applied Analysis, vol. 5, no. 2, pp. 283-293, 1999. 
[27] M. A. Alghamdi, V. Berinde, and N. Shahzad, "Fixed points of multivalued nonself almost contractions," Journal of Applied Mathematics, vol. 2013, Article ID 621614, 6 pages, 2013.

[28] W.-S. Du, E. Karapinar, and N. Shahzad, "The study of fixed point theory for various multivalued non-self-maps," Abstract and Applied Analysis, vol. 2013, Article ID 938724, 9 pages, 2013.

[29] W.-S. Du, "A note on approximate fixed point property and DuKarapinar-Shahzad's intersection theorems," Journal of Inequalities and Applications, vol. 2013, article 506, 2013.

[30] J. Caristi, "Fixed point theorems for mappings satisfying inwardness conditions," Transactions of the American Mathematical Society, vol. 215, pp. 241-251, 1976. 


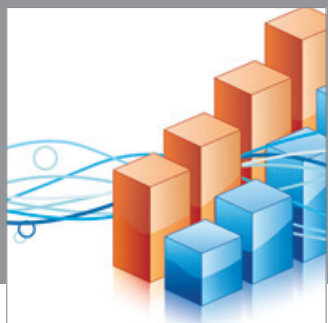

Advances in

Operations Research

mansans

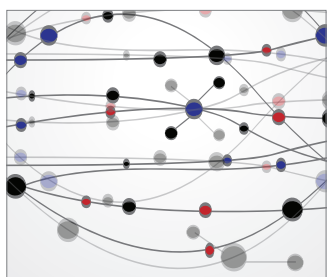

The Scientific World Journal
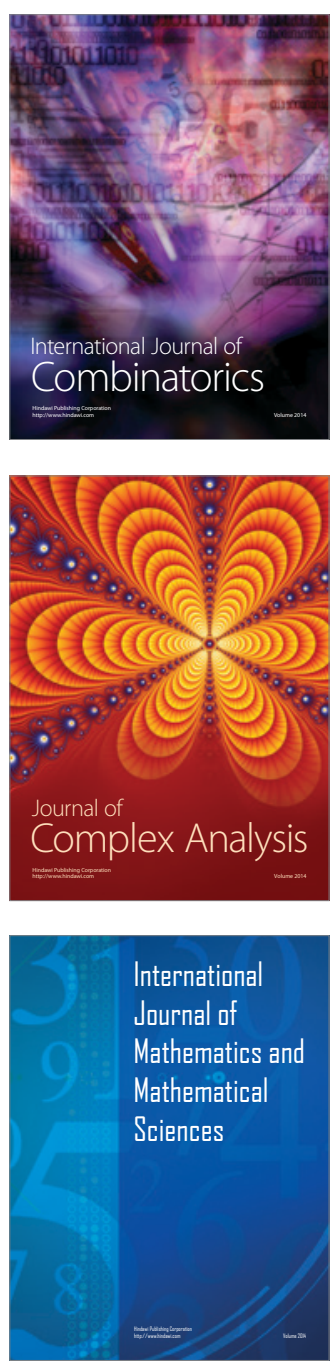
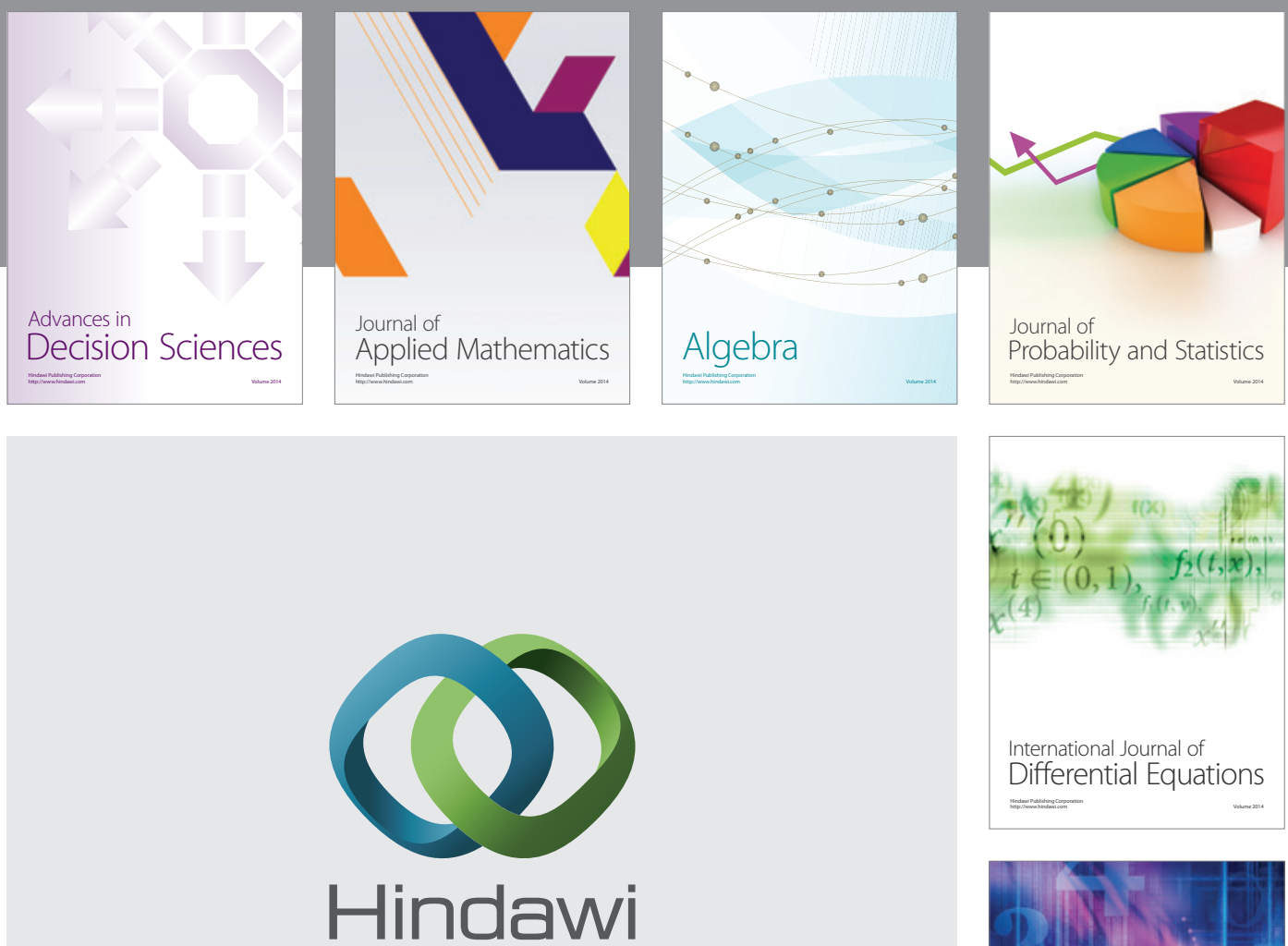

Submit your manuscripts at http://www.hindawi.com
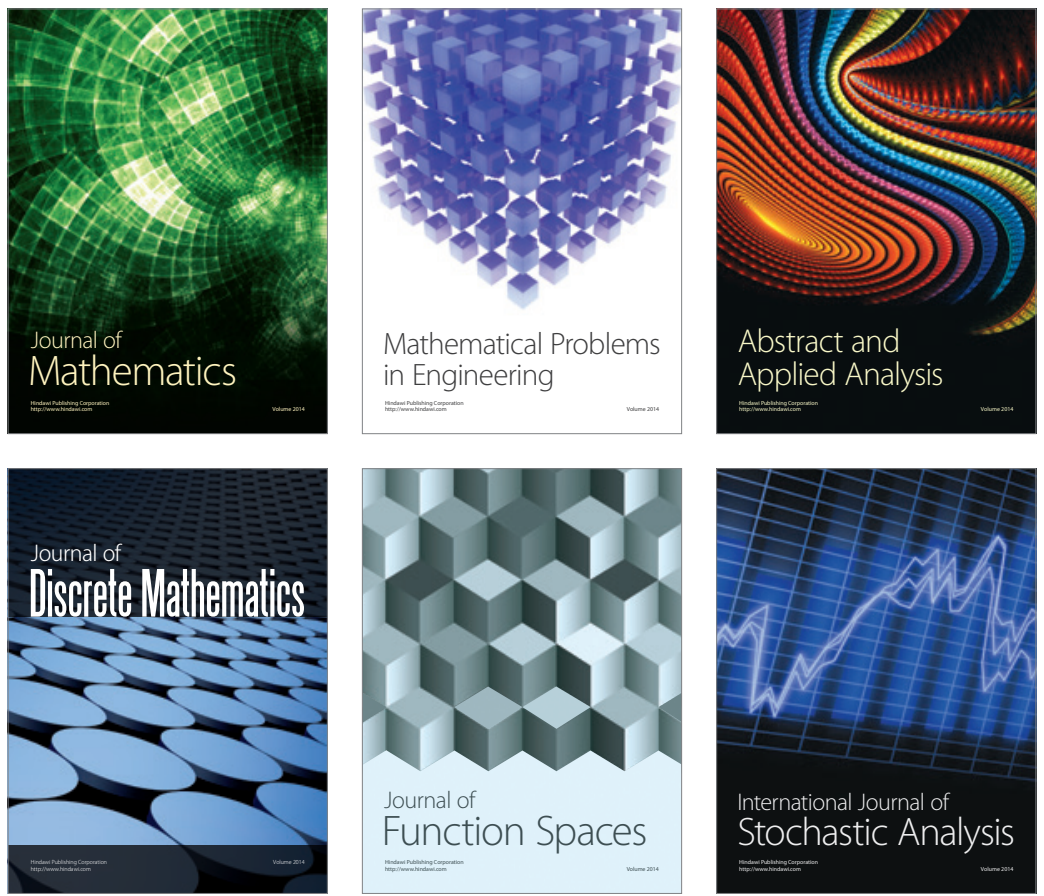

Journal of

Function Spaces

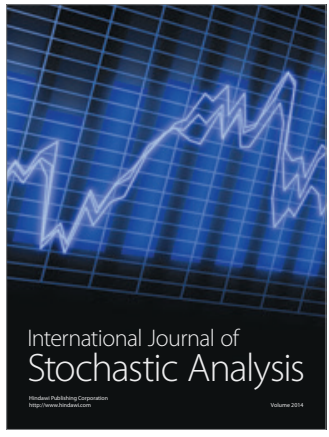

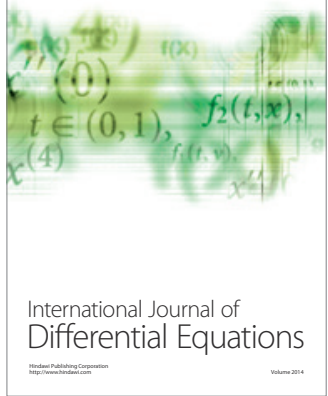
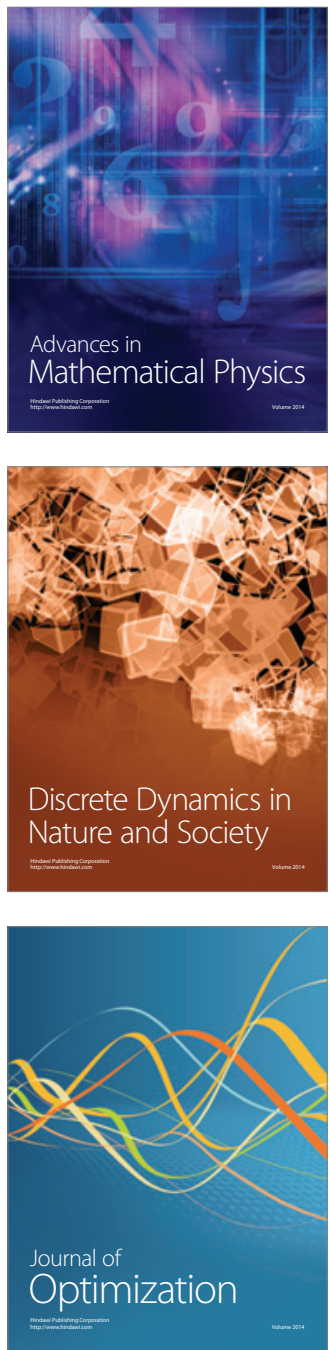